\title{
Fabrication of genistein-loaded biodegradable TPGS-b-PCL nanoparticles for improved therapeutic effects in cervical cancer cells
}

This article was published in the following Dove Press journal:

International Journal of Nanomedicine

27 March 2015

Number of times this article has been viewed

\author{
Hongling Zhang ${ }^{1,2, *}$ \\ Gan Liu'1,2,* \\ Xiaowei Zeng ${ }^{1,2}$ \\ Yanping $\mathrm{Wu}^{1,2}$ \\ Chengming Yang ${ }^{3}$ \\ Lin Mei ${ }^{1,2}$ \\ Zhongyuan Wang 2,4 \\ Laiqiang Huang ${ }^{1,2}$
}

'School of Life Sciences, Tsinghua University, Beijing, People's Republic of China; ${ }^{2}$ The Shenzhen Key

Laboratory of Gene and Antibody

Therapy, Center for Biotechnology and Biomedicine and Division of Life and Health Sciences, Graduate School at Shenzhen, Tsinghua University, Shenzhen, Guangdong, People's Republic of China; ${ }^{3}$ Xili Hospital, Shenzhen, Guangdong, People's Republic of China; ${ }^{4}$ School of Medicine, Shenzhen University, Shenzhen, People's Republic of China

*These authors contributed equally to this work

\begin{abstract}
Genistein is one of the most studied isoflavonoids with potential antitumor efficacy, but its poor water solubility limits its clinical application. Nanoparticles (NPs), especially biodegradable NPs, entrapping hydrophobic drugs have promising applications to improve the water solubility of hydrophobic drugs. In this work, TPGS- $b$-PCL copolymer was synthesized from $\varepsilon$-caprolactone initiated by d- $\alpha$-tocopheryl polyethylene glycol 1000 succinate (TPGS) through ring-opening polymerization and characterized by Fourier transform infrared spectroscopy, proton nuclear magnetic resonance spectroscopy, gel permeation chromatography, and thermogravimetric analysis. The genistein-loaded NPs were prepared by a modified nanoprecipitation method and characterized in the aspects of particle size, surface charge, morphology, drug loading and encapsulation efficiency, in vitro drug release, and physical state of the entrapped drug. The TPGS- $b$-PCL NPs were found to have higher cellular uptake efficiency than PCL NPs. MTT and colony formation experiments indicated that genistein-loaded TPGS- $b$-PCL NPs achieved the highest level of cytotoxicity and tumor cell growth inhibition compared with pristine genistein and genistein-loaded PCL NPs. Furthermore, compared with pristine genistein and genistein-loaded PCL NPs, the genistein-loaded TPGS- $b$-PCL NPs at the same dose were more effective in inhibiting tumor growth in the subcutaneous HeLa xenograft tumor model in $\mathrm{BALB} / \mathrm{c}$ nude mice. In conclusion, the results suggested that genistein-loaded biodegradable TPGS- $b$-PCL nanoparticles could enhance the anticancer effect of genistein both in vitro and in vivo, and may serve as a potential candidate in treating cervical cancer.
\end{abstract}

Keywords: nanomedicine, genistein, TPGS- $b$-PCL, drug delivery, anticancer effect, cytotoxicity

\section{Introduction}

Although great progress has been made in prevention, early diagnosis, and treatment, cancer will remain a leading human health problem, causing an increase in the percentage of morbidity and mortality both in developed and developing countries. Cervical carcinoma is mainly caused by human papillomavirus (HPV) and is the third most common cancer in women all over the world. Because of cancer screening tests and vaccine, cervical cancer has relatively low mortality in Western countries. However, it is still the leading cause of cancer death in women in developing nations. ${ }^{1}$

Many kinds of natural compounds derived from traditional Chinese medicine have been confirmed to have antitumor properties and also have little or no toxicity compared with synthetic chemicals. ${ }^{2}$ Flavonoids, including isoflavones, are natural polyphenolic compounds present ubiquitously in many plants and have antioxidant, anti-inflammatory, and antitumor properties. ${ }^{3,4}$ Genistein $\left(4^{\prime}, 5,7\right.$-trihydroxyisoflavone, Figure 1) is one of the most abundant and best studied soy isoflavones and has received
Correspondence: Zhongyuan Wang; Laiqiang Huang zen Key Laboratory of Gene and Antibody Therapy, Center for Biotechnology and Biomedicine and Division of Life and Health Sciences, Graduate School at Shenzhen, Tsinghua University, L40I, Tsinghua Campus, Xili University Town, Shenzhen, Guangdong 518055, People's Republic of China Email wangzhongyuan@szu.edu.cn; huangl@sz.tsinghua.edu.cn 


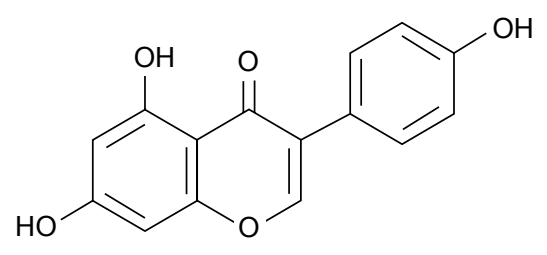

Figure I The molecular structure of genistein.

great attention for its many physiological functions, including potential antitumor activity. ${ }^{5,6}$ Recent studies have indicated that genistein could inhibit tumor cell growth and proliferation, arrest cell cycle at G2/M phase, suppress tumor migration, invasion, and angiogenesis, and induce apoptosis and autophagocytosis. ${ }^{7-11}$ However, the clinical use of genistein for cancer therapy was hindered by its poor water solubility and oral bioavailability. The emerging applications of nanotechnology-based cancer therapy provide a potent platform to improve poor water solubility and bioavailability of hydrophobic antitumor agents. ${ }^{12,13}$

Recently, nanoparticle (NP)-based anticancer drug delivery systems, especially drug formulations with biodegradable polymeric NPs, have attracted considerable attention for their numerous advantages such as high cellular uptake, enhanced permeability and retention effect, and reduced cancer cell drug resistance. ${ }^{14-17}$ Among the US Food and Drug Administration (FDA)-approved biodegradable polymers, poly( $\varepsilon$ caprolactone) (PCL) has gained considerable interest for drug delivery. However, its slow degradation rate and bad compatibility with soft tissues limit its application in nanomedicine. These drawbacks could be solved by copolymerizing PCL with other monomers, such as an amphiphilic water-soluble PEGylated derivative of natural vitamin E (D- $\alpha$-tocopheryl polyethylene glycol 1000 succinate [TPGS]), which is also approved by the FDA. ${ }^{18-21}$ Other than being an excellent emulsifier, TPGS itself has been reported to have anticancer efficacy because of its function as an inhibitor of P-glycoprotein to surmount multidrug resistance in tumor cells. ${ }^{22-24}$

In this work, the TPGS- $b$-PCL copolymer was synthesized and characterized. The genistein-loaded NPs were prepared with a modified nanoprecipitation method instead of solvent extraction/evaporation method and characterized. Compared with the NPs prepared by the solvent extraction/ evaporation method, the current NPs were much smaller. The novel nanoformulations had higher cellular uptake and could accumulate at the tumor site preferentially due to their enhanced permeability and retention effects. Furthermore, this kind of NPs used as drug carriers have other advantages, such as more reasonable pharmacokinetics and more desirable biodistribution as well as easy industry application. ${ }^{15,25}$ Also, the efficacy of genistein-loaded TPGS- $b$-PCL NPs on human cervical carcinoma was investigated both in vitro and in vivo, in close comparison with pristine genistein and genisteinloaded PCL NPs. These results suggest that genistein-loaded TPGS- $b$-PCL nanoformulation has potential application in human cervical cancer therapy.

\section{Materials and methods Materials}

PCL (MW, 14,000 Da), $\varepsilon$-caprolactone ( $\varepsilon$-CL), TPGS, stannous octoate $\left(\mathrm{Sn}(\mathrm{Oct})_{2}\right)$, methanol (HPLC [high-performance liquid chromatography]-grade), genistein powder with about $98 \%$ purity (HPLC-grade), coumarin-6, 3-(4,5-dimethylthiazol2-yl)-2,5-diphenyltetrazolium bromide (MTT), and 4',6diamidino-2-phenylindole (DAPI) were purchased from Sigma-Aldrich (St Louis, MO, USA). Dulbecco's Modified Eagle's Medium (DMEM) and fetal bovine serum were obtained from Gibco BRL (Invitrogen Co, Carlsbad, CA, USA). Human cervical carcinoma cell line HeLa cells were kept by our laboratory.

\section{Synthesis and characterization of TPGS- $b$ - PCL copolymer}

Synthesis of TPGS- $b$-PCL copolymer was carried out through ring-opening polymerization, as previously described. ${ }^{18,26}$ The reaction route scheme is shown in Figure 2. In brief, $\varepsilon$-CL (1.63 g, $14.3 \mathrm{mmol})$, TPGS (0.15 g, $0.1 \mathrm{mmol})$, and catalyst $\mathrm{Sn}(\mathrm{Oct})_{2}(0.02 \mathrm{~g}, 8 \mu \mathrm{L})$ were added in a dried flask, which was connected to a vacuum system, evacuated, refilled with nitrogen gas, and sealed. Then the flask was heated in an oil bath at $160^{\circ} \mathrm{C}$ and the mixture was allowed to react overnight. After cooling to room temperature, the product was dissolved in dichloromethane and then precipitated in excess cold methanol to remove any impurity. Thereafter, the crude product was collected by filtration and washed twice with methanol to remove the oligomer and unreacted monomer. Finally, the synthetic TPGS- $b$-PCL copolymer was dried under vacuum at $40^{\circ} \mathrm{C}$ for 24 hours.

The molecular structure of TPGS- $b$-PCL copolymer was investigated using Fourier transform infrared (FTIR) spectrophotometer (Thermo Nicolet; Thermo Fisher Scientific, Waltham, MA, USA). The structure of synthesized TPGS- $b$ PCL copolymer was verified by a nuclear magnetic resonance ( ${ }^{1} \mathrm{H}-\mathrm{NMR}$ ) spectrometer (Bruker ACF 300; Bruker Instruments Inc., Billerica, MA, USA) using $\mathrm{CDCl}_{3}$ as a solvent. Gel permeation chromatography (GPC) was carried out to determine the weight-averaged molecular weight and weight 
<smiles>Cc1c(C)c2c(c(C)c1OC(=O)CC(=O)OCCO)CCC(C)(CCCC(C)CCCC(C)CCCC(C)C)O2</smiles>

$\varepsilon-C L$ monomer

TPGS

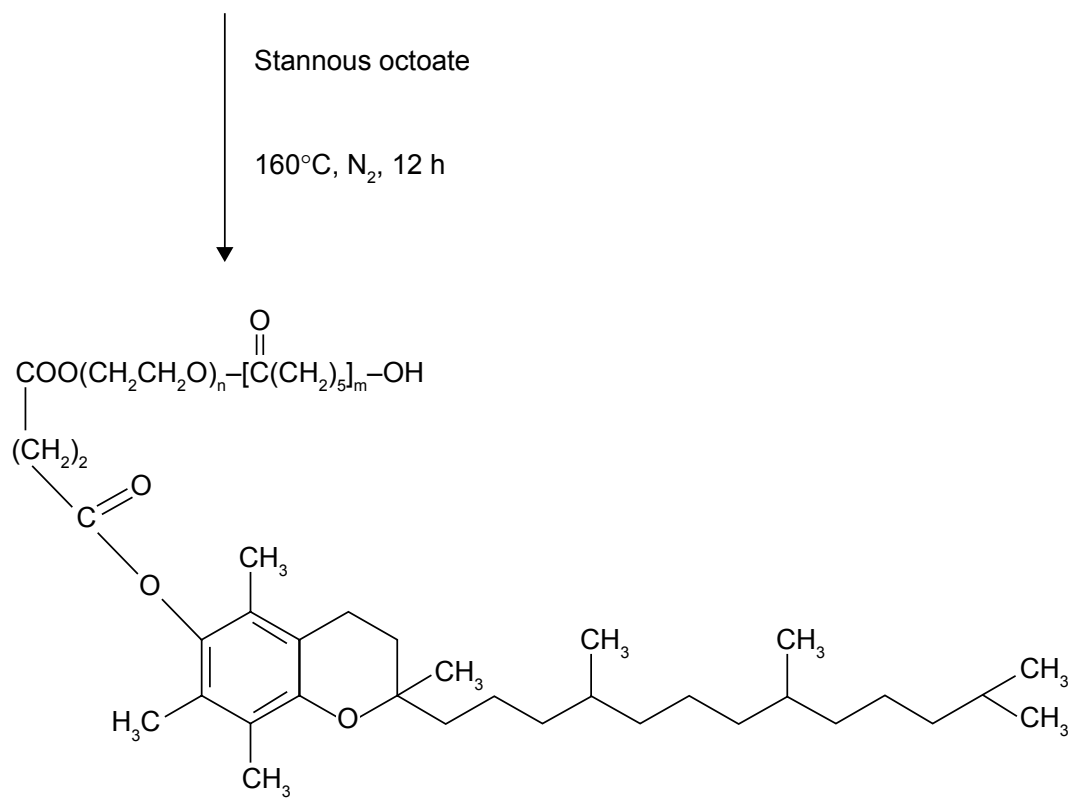

TPGS-b-PCL copolymer

Figure 2 Schematic description of the synthesis of TPGS-b-PCL diblock copolymer.

Abbreviations: $\varepsilon-C L$, $\varepsilon$-caprolactone; h, hours; PCL, poly( $\varepsilon$-caprolactone); TPGS, D- $\alpha$-tocopheryl polyethylene glycol 1000 succinate.

distribution of the copolymer. The thermal property of the TPGS- $b$-PCL copolymer was measured by thermogravimetric analysis (TGA; TGA 2050 thermogravimetric analyzer, PerkinElmer Inc., Waltham, MA, USA).

\section{Formulation and characterization of genistein-loaded nanoparticles}

Genistein-loaded PCL and TPGS- $b$-PCL NPs were prepared by a modified nanoprecipitation method, as previously described. ${ }^{26,27} \mathrm{In}$ short, $10 \mathrm{mg}$ of genistein and $100 \mathrm{mg}$ of PCL polymer or TPGS- $b$-PCL copolymer were dissolved in $8 \mathrm{~mL}$ of acetone. Then the dissolved mixture was slowly added into $100 \mathrm{~mL}$ of $0.03 \%$ (w/v) TPGS aqueous solution using a $1 \mathrm{~mL}$ injector, under stirring. After stirring overnight at room temperature to completely remove acetone, the suspension was centrifuged at $20,000 \times g$ for 15 minutes and then washed three times to remove the emulsifier TPGS and unencapsulated genistein. Finally, the resulting NPs were dispersed in $10 \mathrm{~mL}$ of water and lyophilized for further use. The preparation of fluorescent coumarin-6-loaded PCL and TPGS- $b$-PCL NPs was by the same method except for $1 \mathrm{mg}$ of coumarin- 6 instead of genistein.

The particle size, size distribution, and zeta potential of NPs were determined by dynamic light scattering (Zetasizer Nano ZS90; Malvern Instruments, Malvern, UK). All measurements were taken at $25^{\circ} \mathrm{C}$. The morphology of genistein-loaded TPGS- $b$-PCL NPs was observed by a field emission scanning electron microscopy (FESEM) using a JEOL JSM-6301F (JEOL, Tokyo, Japan) system operated at a $5.0 \mathrm{kV}$ accelerating voltage and further examined by transmission electron microscopy (TEM; Tecnai G2 20, FEI Company, Hillsboro, OR, USA). 
Drug loading (DL) and encapsulation efficiency (EE) of genistein-loaded PCL NPs and TPGS- $b$-PCL NPs were determined using HPLC (LC 1200, Agilent Technologies, Santa Clara, CA, USA) as follows. Briefly, $5 \mathrm{mg}$ of lyophilized genistein-loaded NPs was dissolved in $100 \mu \mathrm{L}$ of dichloromethane and then diluted with methanol. After passing through a $0.45 \mu \mathrm{m}$ filter to remove precipitation, the clear solution was subjected to HPLC analysis using a reverse-phase C-18 column $(150 \times 4.6 \mathrm{~mm}$; pore size, $5 \mu \mathrm{m}$; Agilent Technologies) at $25^{\circ} \mathrm{C}$ to determine the amount of encapsulated genistein at $262 \mathrm{~nm}$. Methanol/water (60/40, $\mathrm{v} / \mathrm{v}$ ) was used as the mobile phase at a flow rate of $1 \mathrm{~mL} / \mathrm{min}$. The amount of genistein was calculated according to a standard genistein sample. The DL and EE of genistein in NPs were calculated as follows:

$$
\begin{gathered}
\text { DL } \%=\frac{\text { Actual amount of genistein in NPs }}{\text { Amount of NPs }} \times 100 \% \\
\mathrm{EE} \%=\frac{\text { Actual DL }}{\text { Theoretical DL }} \times 100 \%
\end{gathered}
$$

Differential scanning calorimetry (DSC Q2000 thermogravimetric analyzer, PerkinElmer Inc.) was carried out to determine the physical status of genistein inside the NPs. The pristine genistein, blank NPs, or genistein-loaded NPs were purged with dry nitrogen at a flow rate of $20 \mathrm{~mL} / \mathrm{min}$, and the temperature was raised at a rate of $10^{\circ} \mathrm{C} / \mathrm{min}$.

In vitro drug release profiles of genistein-loaded PCL and TPGS- $b$-PCL NPs were investigated by a dialysis method as described previously. ${ }^{18,28}$ In brief, $10 \mathrm{mg}$ of lyophilized genistein-loaded NPs was resuspended in $1 \mathrm{~mL}$ of releasing buffer (PBS [phosphate-buffered saline] containing $0.1 \% \mathrm{w} / \mathrm{v}$ Tween-80) and transferred into a dialysis tube ( $\mathrm{MWCO}=3.5 \mathrm{kDa}$ and the dialysis area is $1 \mathrm{~cm}^{2}$ ). Subsequently, the dialysis tubes were immersed into a centrifuge tube containing $10 \mathrm{~mL}$ of releasing buffer and incubated at $37^{\circ} \mathrm{C}$ with gentle shaking $(120 \times g)$. At designated time intervals, the entire release buffer was collected and replaced with prewarmed fresh release buffer. The released genistein was quantified using HPLC in terms of DL and EE.

\section{Cell culture}

Human cervical carcinoma cell line HeLa cells were cultured in DMEM supplemented with $10 \%$ fetal bovine serum, $100 \mathrm{U} / \mathrm{mL}$ penicillin, and $100 \mathrm{mg} / \mathrm{mL}$ streptomycin at $37^{\circ} \mathrm{C}$ in a humidified atmosphere containing $5 \%$ carbon dioxide.

\section{Cellular uptake of NPs}

Because coumarin- 6 could serve as fluorescence, it was encapsulated into PCL NPs and TPGS- $b$-PCL NPs to quantitatively and qualitatively evaluate the cellular uptake of NPs. ${ }^{29-31}$

For quantification of PCL NPs and TPGS- $b$-PCL NPs uptake by HeLa cells, the cells were seeded in a 96-well black culture plate at an initial density of $2 \times 10^{4}$ cells/well and cultured overnight. Then the medium was removed and incubated with fresh medium containing 100, 250, and $500 \mu \mathrm{g} / \mathrm{mL}$ coumarin-6-loaded PCL NPs or TPGS- $b$-PCL NPs for further $2 \mathrm{~h}$. Subsequently, the cells were washed and lysed with $50 \mu \mathrm{L}$ of $0.2 \mathrm{~N} \mathrm{NaOH}$ (containing $0.5 \%$ Triton $\mathrm{X}-100$ ), and the fluorescence intensity was determined by a microplate reader (GENios, Tecan, Switzerland) with excitation wave length at $430 \mathrm{~nm}$ and emission wave length at $485 \mathrm{~nm}$.

For qualitative observation, HeLa cells were seeded onto coverslips in a six-well plate at a density of $2 \times 10^{5}$. After 24 hours of attachment, the medium was replaced with fresh medium containing coumarin-6-loaded NPs at a concentration of $250 \mu \mathrm{g} / \mathrm{mL}$ for 4 hours. Then the cells were washed three times with cold PBS, fixed by cold methanol for 20 minutes, and further washed twice with PBS, and the nuclei were counterstained with DAPI for 10 minutes. Finally, the cells were mounted on microscope slides using fluorescent mounting medium and imaged using a confocal laser scanning microscope (Olympus Fluoview FV-1000, Tokyo, Japan).

\section{Cytotoxicity assay}

The in vitro cytotoxicity assay of pristine genistein, genisteinloaded PCL NPs, and TPGS- $b$-PCL NPs were performed on HeLa cells using the MTT method. In brief, HeLa cells were plated in 96-well transparent plates at an initial density of $2 \times 10^{4}$ cells/well. After 24 hours of attachment, the medium was replaced with fresh medium containing genistein-loaded NP suspensions or pristine genistein at final concentrations of 5.0, 10.0, 20.0, and $40.0 \mu \mathrm{g} / \mathrm{mL}$ equivalent genistein or drug-free NP suspension with the same amount of NPs for 24, 48, and 72 hours. At the designed time intervals, the cell viability was measured using MTT assay standard protocol at $570 \mathrm{~nm}$ using a microplate reader (Bio-Rad Model 680; Bio-Rad Laboratories Inc., Hertfordshire, UK). $\mathrm{IC}_{50}$, the drug concentration at which $50 \%$ of cell growth is inhibited, was measured at final concentrations of 5.0, 10.0, 20.0, 30.0, $40.0,60.0$, and $80.0 \mu \mathrm{g} / \mathrm{mL}$ equivalent genistein for 24,48 , and 72 hours. 


\section{Colony formation analysis}

HeLa cells were cultured in a six-well plate at an initial density of 500 cells/well and were allowed to attach for 24 hours. After preincubation, the medium was incubated with fresh medium or fresh medium containing genistein-loaded PCL or TPGS- $b$-PCL $\mathrm{NP}$ suspensions or pristine genistein at final concentrations of 10 $\mu \mathrm{g} / \mathrm{mL}$ equivalent genistein or drug-free $\mathrm{NP}$ suspension with the same amount of NPs for 48 hours. Then, the cells were washed three times with prewarmed PBS and recultured in fresh DMEM for another 10 days. Finally, the cells were stained with crystal violet and images were captured using a digital camera.

\section{In vivo anticancer effect}

All animal studies were carried out according to the protocols approved by the Administrative Committee on Animal Research in Graduate School at Shenzhen, Tsinghua University. Fiveweek-old female BALB/c nude mice were purchased from the Institute of Laboratory Animal Sciences, Chinese Academy of Medical Science. They were subcutaneously implanted with $7.5 \times 10^{6} \mathrm{HeLa}$ cells/mouse $/ 100 \mu \mathrm{L}$ at the right flank region. After inoculation of the HeLa cells, the subcutaneous tumor in each mouse was closely monitored. The tumor volume can be calculated from the formula: Tumor volume $\left(\mathrm{mm}^{3}\right)=0.524 \times$ (length) $\times(\text { width })^{2}$. When the tumor was touchable, the mice were divided into three groups (five mice per group) and the in vivo antitumor studies were performed. The HeLa tumorbearing female BALB/c nude mice were intraperitoneally injected with saline (as a control), the genistein-loaded PCL and TPGS- $b$-PCL nanoformulations, or pristine genistein, at a dose of $50 \mathrm{mg} / \mathrm{kg}$ equivalent genistein every other day. Animal body weights and tumor volumes were measured every other day. After treatment for 16 days, the animals were humanely killed by cervical decapitation and tumors were excised and weighted. The terminal tumor weight (in milligram) was used to evaluate the anticancer activity of genistein-loaded TPGS- $b$-PCL NPs.

\section{Statistical analysis}

Data were expressed as the mean value \pm standard deviation (SD). Statistical analysis was done with one-way analysis of variance (ANOVA) using the SPSS software. $P$-values $<0.05$ were considered to be statistically significant.

\section{Results and discussion}

\section{Synthesis and characterization of TPGS-b- PCL copolymer}

The TPGS- $b$-PCL copolymer used in this study was prepared through the ring-opening polymerization of $\varepsilon$-CL initiated by
TPGS by using $\left(\mathrm{Sn}(\mathrm{Oct})_{2}\right)$ as the catalyst. Figure 2 schematically illustrates the general synthetic route.

The FTIR of the TPGS- $b$-PCL copolymer and TPGS are presented in Figure 3. The carbonyl band of TPGS appears at $1,739 \mathrm{~cm}^{-1}$. For the synthesized diblock copolymer, the carbonyl band is shifted to about $1,730 \mathrm{~cm}^{-1}$, which is different from the carbonyl bands of PCL at $1,725-1,726 \mathrm{~cm}^{-1} .18,32$ In the TPGS$b$-PCL spectrum, the bands in the range $2,867-2,949 \mathrm{~cm}^{-1}$ are assigned to $-\mathrm{CH}_{2}$ stretching band of $\mathrm{PCL}$, and the $\mathrm{CH}_{2}$ stretching band of TPGS at $2,880 \mathrm{~cm}^{-1}$ was not observed because of overlapping with that of TPGS- $b$-PCL copolymer. The absorption band at about $3,442 \mathrm{~cm}^{-1}$ is due to the terminal hydroxyl group and that at $1,045-1,297 \mathrm{~cm}^{-1}$ is attributed to the $\mathrm{C}-\mathrm{O}$ stretching. Of those, the absorption band at $1,242 \mathrm{~cm}^{-1}$ is assigned to asymmetric COC stretching. The band at about $1,297 \mathrm{~cm}^{-1}$ has been used for the investigation of the crystallinity change in PCL.

${ }^{1} \mathrm{H}-\mathrm{NMR}$ was carried out to determine the TPGS content, molecular weight, and structure of the obtained TPGS- $b$ PCL copolymer, and the results are presented in Figure 4. Peaks at 1.39, 1.65, 2.29-2.33, and $4.06 \mathrm{ppm}$ are assigned to $-\mathrm{CH}_{2}$ protons in PCL units. ${ }^{18,32}$ The peak at $3.65 \mathrm{ppm}$ was assigned to the $-\mathrm{CH}_{2}$ protons of poly(ethylene oxide) part of TPGS. The lower peaks in the aliphatic region belong to various moieties of vitamin $\mathrm{E}$ tails. The number-averaged molecular weight $\left(M_{\mathrm{n}}\right)$ of the TPGS- $b$-PCL copolymer could be calculated by using the ratio between the peak areas at 3.65 and $4.06 \mathrm{ppm}$. The $M_{\mathrm{n}}$ of the TPGS- $b$-PCL copolymer was determined to be 18,473 . The ratio of $\varepsilon$-CL and TPGS

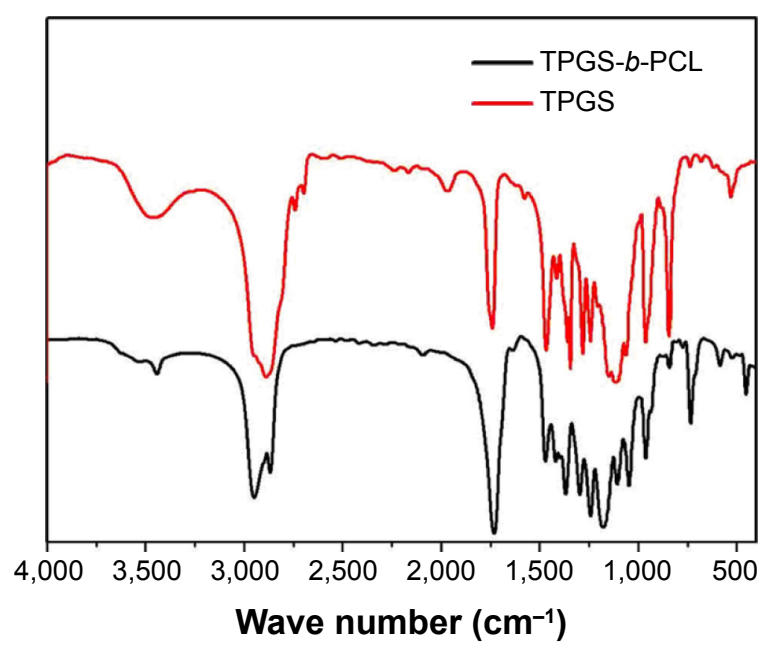

Figure 3 FTIR spectra of TPGS and TPGS-b-PCL copolymer.

Abbreviations: FTIR, Fourier transform infrared; PCL, poly( $\varepsilon$-caprolactone); TPGS, D- $\alpha$-tocopheryl polyethylene glycol 1000 succinate. 
A

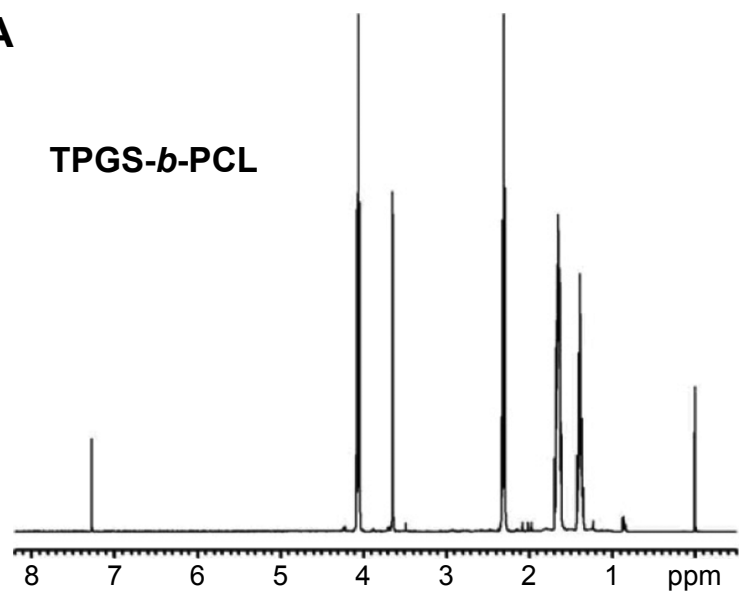

B

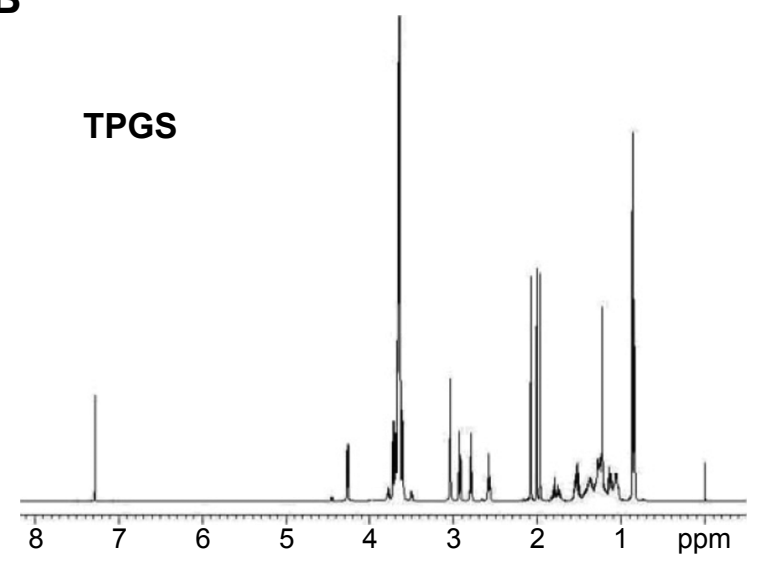

Figure 4 Typical 'H-NMR spectra of TPGS-b-PCL copolymer (A) and TPGS (B).

Abbreviations: 'H-NMR, proton nuclear magnetic resonance; PCL, poly( $\varepsilon$-caprolactone); TPGS, D- $\alpha$-tocopheryl polyethylene glycol 1000 succinate.

molecular mass which were integrated into the TPGS- $b$-PCL copolymer is $91.81 \%$ and $8.19 \%$.

In the GPC analysis (Figure 5A), a monomodal pattern was observed, indicating that the polymer product is a pure copolymer not contaminated by PCL and TPGS. The peak for TPGS appeared at 28.3 minutes. Instead, the peak of the TPGS- $b$-PCL copolymer shifted to 17.9 minutes. The polydispersity of the copolymer molecular weight was about 1.08 . The $M_{\mathrm{n}}$ calculated from the GPC chromatograph was 20,791 , which is in good agreement with the results of ${ }^{1} \mathrm{H}-\mathrm{NMR}$ studies.

To investigate the thermal properties of the synthesized copolymer, TGA was performed. As shown in Figure 5B, there are two typical stages of weight loss for TPGS- $b$ PCL diblock copolymer, whereas the TPGS monomer has only a single step of weight loss. Each turning point marked the combustion of a new component in the copolymer. ${ }^{18,32}$

\section{Preparation and characterization of genistein-loaded PCL and TPGS-b-PCL NPs}

Genistein was encapsulated by PCL and TPGS- $b$-PCL to improve the drug's poor water solubility. As described previously, ${ }^{26,27}$ genistein-loaded PCL and TPGS- $b$-PCL NPs were prepared by a modified nanoprecipitation method using acetone as the solvent and TPGS as the emulsifier. Then the NPs were characterized in detail.

\section{Particle size, size distribution, and zeta potential}

As reported previously, the NP size and surface chemistry influence absorption, biodistribution, and pharmacokinetics of
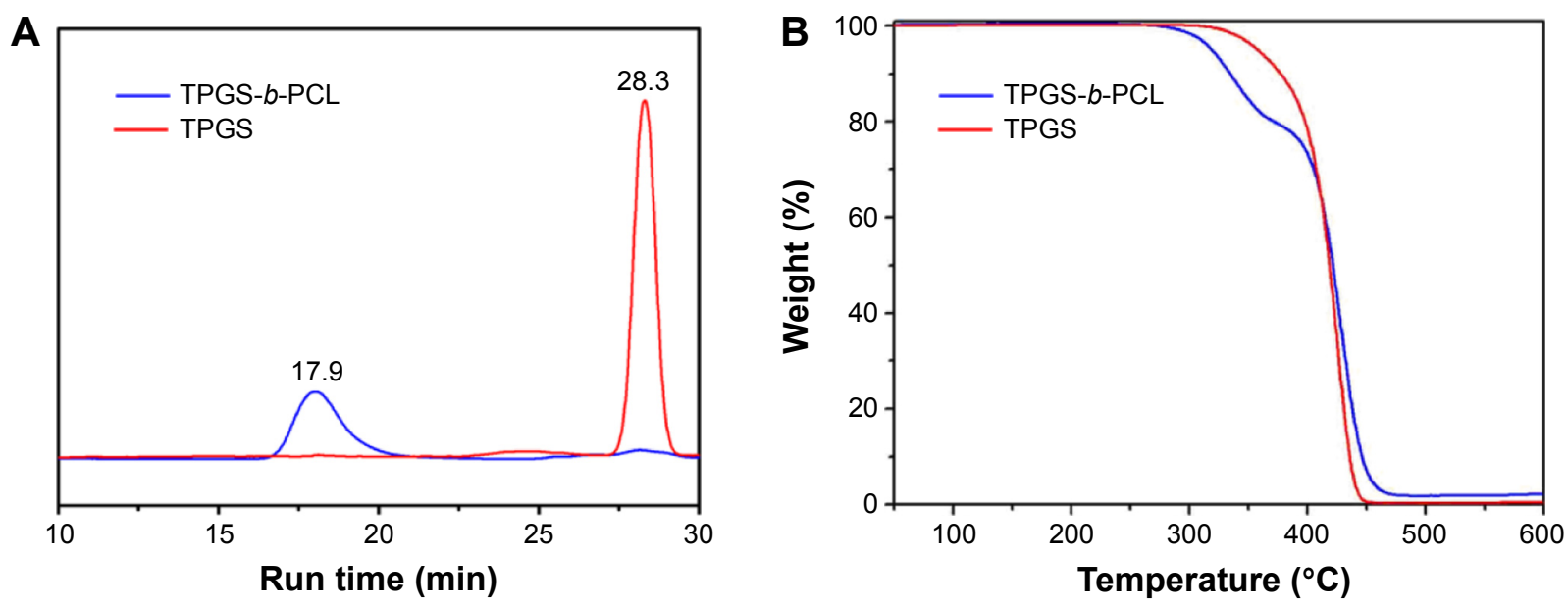

Figure 5 Characterization of TPGS-b-PCL copolymer.

Notes: (A) Typical gel permeation chromatograms of TPGS and TPGS-b-PCL diblock copolymer; (B) thermogravimetric profiles of TPGS and TPGS-b-PCL copolymer. Abbreviations: min, minutes; PCL, poly( $\varepsilon$-caprolactone); TPGS, D- $\alpha$-tocopheryl polyethylene glycol 1000 succinate. 
Table I Characterization of nanoparticles

\begin{tabular}{lllllll}
\hline Group & $\begin{array}{l}\text { Size }(\mathbf{n m}) \\
(\mathbf{n}=\mathbf{5})\end{array}$ & $\begin{array}{l}\text { Polydispersion } \\
(\mathbf{n}=\mathbf{5})\end{array}$ & $\begin{array}{l}\text { ZP }(\mathbf{m V}) \\
(\mathbf{n}=\mathbf{5})\end{array}$ & $\begin{array}{l}\text { Theoretical drug } \\
\text { loading (\%) }\end{array}$ & $\begin{array}{l}\text { Drug loading (\%) } \\
(\mathbf{n}=\mathbf{5})\end{array}$ & $\begin{array}{l}\text { Encapsulation efficiency (\%) } \\
(\mathbf{n}=\mathbf{5})\end{array}$ \\
\hline PCL NPs & $225.80 \pm 3.52$ & $0.291 \pm 0.007$ & $-11.29 \pm 1.43$ & 9.09 & $8.21 \pm 0.25$ & $90.27 \pm 2.72$ \\
TPGS-b-PCL NPs & $181.83 \pm 2.76$ & $0.255 \pm 0.005$ & $-14.70 \pm 1.74$ & 9.09 & $8.69 \pm 0.29$ & $95.56 \pm 3.24$
\end{tabular}

Abbreviations: NPs, nanoparticles; PCL, poly( $\varepsilon$-caprolactone); TPGS, D- $\alpha$-tocopheryl polyethylene glycol I000 succinate; ZP, zeta potential.

the drug. ${ }^{33-35}$ As shown in Table 1, the size, size distribution, and zeta potential of NPs were measured using the dynamic light scattering. The mean particle size of genistein-loaded TPGS- $b$-PCL NPs was $181.83 \mathrm{~nm}$, which was much smaller than genistein-loaded PCL NPs (225.80 nm). Moreover, the size distribution of TPGS- $b$-PCL nanoformulation (polydispersity index $[\mathrm{PDI}]=0.255$ ) was a little narrower than that of PCL NPs (PDI =0.291). This is because TPGS is an excellent emulsifier and the TPGS in the TPGS- $b$-PCL copolymer may play a self-emulsifying function, reducing surface energy of the particles to resist coalescence and flocculation of the particles. ${ }^{36,37}$ Zeta potential represents the surface charge of the NP and is a crucial parameter reflecting the stability of NP suspensions. The high magnitude of zeta potential suggests strong electrostatic repellent interactions between NPs and thus sufficient dispersion stability. ${ }^{31,38}$ As shown in Table 1, the zeta potentials of genistein-loaded PCL and
TPGS- $b$-PCL formulations were -11.29 and $-14.70 \mathrm{mV}$, respectively. Compared with positive surface charge, the negative surface charge of both NPs not only indicated the high stability of NP suspensions but also suggested less toxicity to normal cells.

\section{Drug loading and encapsulation efficiency}

The DL and EE, detected by HPLC, are shown in Table 1. The percentage of genistein loaded in the PCL and TPGS$b$-PCL NPs were $8.21 \%$ and $8.69 \%$, respectively. And the encapsulation of the PCL NPs was $90.27 \%$ and that of the TPGS- $b$-PCL NPs was $95.56 \%(\mathrm{n}=5)$. It could be seen that TPGS- $b$-PCL NPs achieved a higher drug EE.

\section{Surface morphology}

A high-resolution image of genistein-loaded TPGS- $b$-PCL NPs was obtained using FESEM and TEM. As shown
A

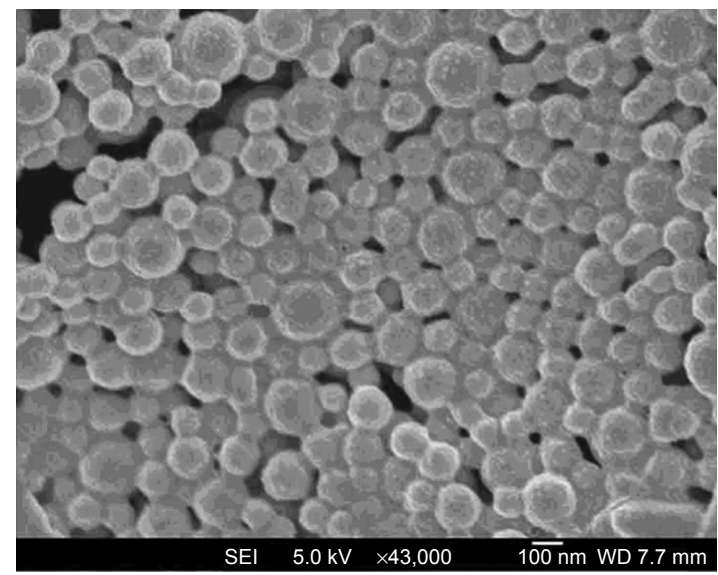

B

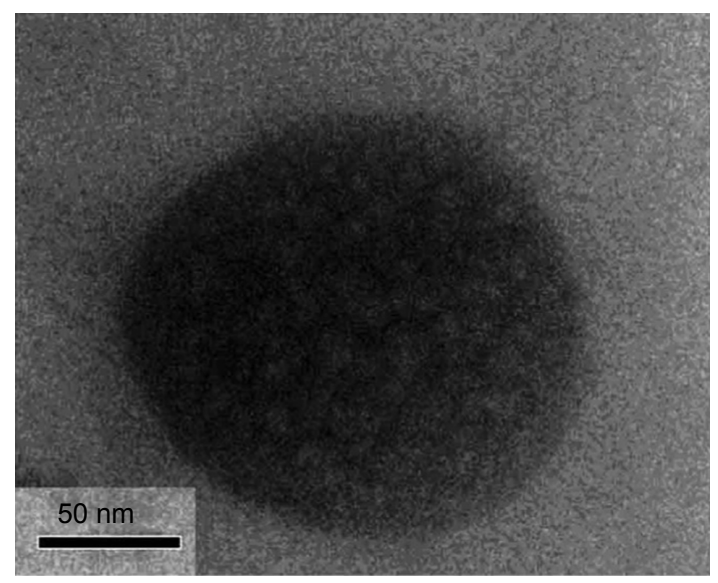

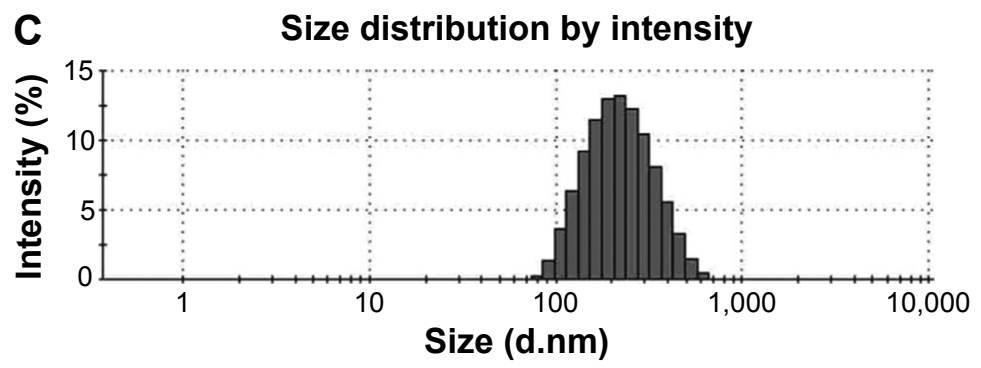

Figure 6 FESEM image (A), TEM image (B), and DLS spectra (C) of genistein-loaded TPGS-b-PCL NPs.

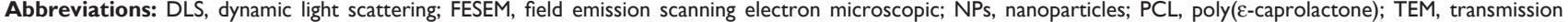
electron microscopic; TPGS, D- $\alpha$-tocopheryl polyethylene glycol 1000 succinate. 
in Figure 6A (FESEM image) and 6B (TEM image), the genistein-loaded TPGS- $b$-PCL NPs were in moderate uniformity with a nearly spherical shape and smooth surface. Moreover, the particle size was around $170 \mathrm{~nm}$, which was slightly smaller than that obtained from the dynamic light scattering method (Figure 6C) because of lack of hydration shell when particle size was determined by FESEM and TEM.

\section{Physical state of genistein in the NPs}

To investigate the physical state of genistein in the NPs, DSC analysis was carried out using pure genistein, genisteinloaded PCL NPs, and genistein-loaded TPGS- $b$-PCL NPs. As shown in Figure 7, the DSC curve of pure genistein indicated a crystalline state with a melting endothermic peak at $301.7^{\circ} \mathrm{C}$. However, when genistein was encapsulated into PCL and TPGS- $b$-PCL NPs, such a peak disappeared, implying that genistein was essentially in an amorphous or disordered crystalline phase after having been encapsulated by both NPs.

\section{In vitro drug release of genistein from PCL NPs and TPGS- $b$-PCL NPs}

Figure 8 illustrates the in vitro drug release profiles of genistein-loaded PCL NPs and TPGS- $b$-PCL NPs in PBS buffer ( $\mathrm{pH} 7.4$ ) supplemented with $0.1 \% \mathrm{w} / \mathrm{v}$ Tween 80 in the first 15 days, which exhibited biphasic release patterns. It could be observed from Figure 8 that the release of genistein from the drug-loaded PCL NPs and TPGS- $b$-PCL
NPs showed an initial burst of $11.97 \%$ and $14.58 \%$, respectively, in the 1 st day. In the following days, the cumulative release of genistein persistently increased. After 15 days' incubation, the cumulative release of genistein was $48.95 \%$ for PCL NPs and $58.84 \%$ for TPGS- $b$-PCL NPs. It could be easily concluded that TPGS- $b$-PCL NPs exhibited a much faster drug release than did PCL NPs. This is probably because of the hydrophilic part of the TPGS, which promotes the uptake and permeation of PBS buffer into the core of NPs to facilitate drug release.

\section{In vitro cellular uptake of NPs}

The internalization of PCL and TPGS- $b$-PCL NPs into cells was determined by measuring the cellular uptake of NPs in vitro. A fluorescent marker coumarin-6-loaded PCL NPs and TPGS- $b$-PCL NPs were used to quantitatively and qualitatively investigate in vitro cellular uptake of NPs. Human cervical carcinoma cell line HeLa was chosen. After incubating HeLa cells with 100, 250, and $500 \mu \mathrm{g} / \mathrm{mL}$ coumarin6 -loaded PCL NPs or TPGS- $b$-PCL NPs for 2 hours, the cellular uptake efficiency of both NPs was measured. As shown in Figure 9A, it could be concluded that the cellular uptake efficiency of NPs decreased with the increasing concentration of NPs, which indicated a saturated and limited capability of cellular uptake of the nanoparticles. Moreover, the TPGS- $b$-PCL NPs exhibited 1.25-, 1.22-, 1.28-fold higher cellular uptake efficiency than did PCL NPs at the concentrations of 100,250 , and $500 \mu \mathrm{g} / \mathrm{mL}$, respectively.

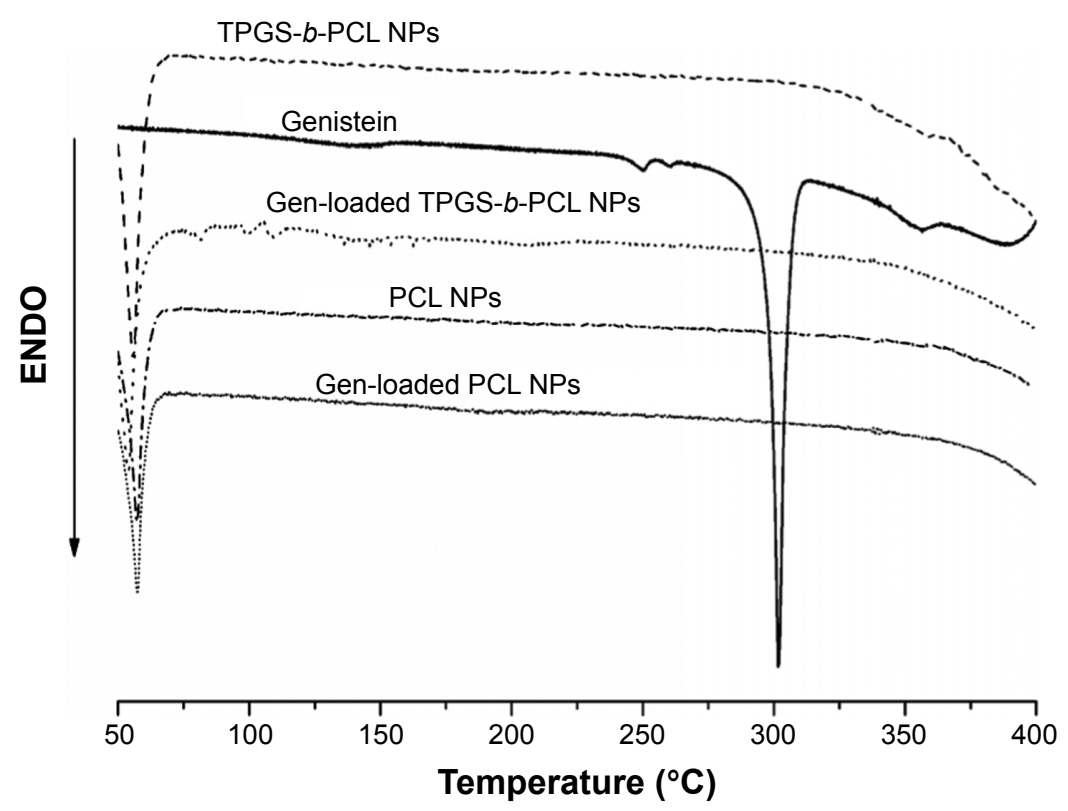

Figure 7 DSC thermograms of the pure genistein, blank PCL NPs, blank TPGS-b-PCL NPs, and genistein-loaded PCL NPs, and TPGS-b-PCL NPs. Abbreviations: DSC, differential scanning calorimetry; ENDO, endotherm; NPs, nanoparticles; PCL, poly( $\varepsilon$-caprolactone); TPGS, D- $\alpha$-tocopheryl polyethylene glycol I000 succinate. 


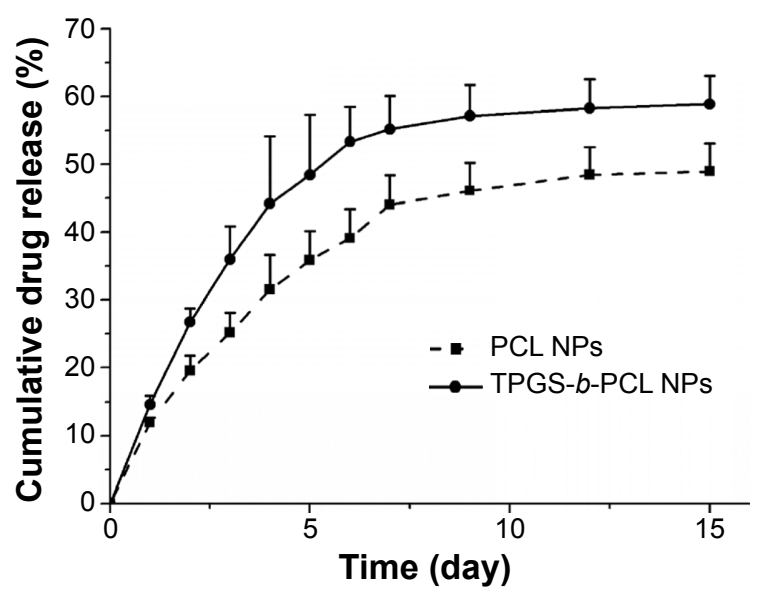

Figure 8 In vitro release profiles of genistein-loaded PCL NPs and TPGS- $b-P C L$ NPs. Abbreviations: NPs, nanoparticles; PCL, poly( $\varepsilon$-caprolactone); TPGS, D- $\alpha$-tocopheryl polyethylene glycol 1000 succinate.

The cellular uptake of NPs was further visualized by confocal laser scanning microscopy. Figure 9B shows the confocal images of HeLa cells after $4 \mathrm{~h}$ of incubation with $250 \mu \mathrm{g} / \mathrm{mL}$ coumarin-6-loaded TPGS- $b$-PCL NPs. The image in column 1 was from DAPI channel (blue) representing the nucleus; the image in column 2, obtained from fluorescein isothiocyanate channel (green), was green fluorescence representing coumarin-6; and the image in column 3 was the merged channels of fluorescein isothiocyanate and DAPI. It could be easily seen from column 3 that most internalized coumarin-6-loaded NPs were in the cytoplasm, although some NPs went into the nucleus, especially into the nucleolus.

\section{In vitro cytotoxicity and cell colony formation analysis}

HeLa cells were used to evaluate the cytotoxicity of pristine genistein, genistein-loaded PCL and TPGS- $b$-PCL NPs, and drug-free PCL and TPGS- $b$-PCL NPs by the MTT method. After 24, 48, and 72 hours incubation with pristine genistein or genistein-loaded PCL NPs and TPGS- $b$-PCL NPs suspension at 5.0,10.0, 20.0, and $40.0 \mu \mathrm{g} / \mathrm{mL}$ equivalent genistein concentrations or drug-free NP suspension with the same amount of NPs, the cell viabilities are shown in Figure 10A-C. It could be concluded that 1) the placebo NPs of PCL and TPGS- $b$-PCL are biocompatible since they show little decrease in cellular viability of HeLa cells;
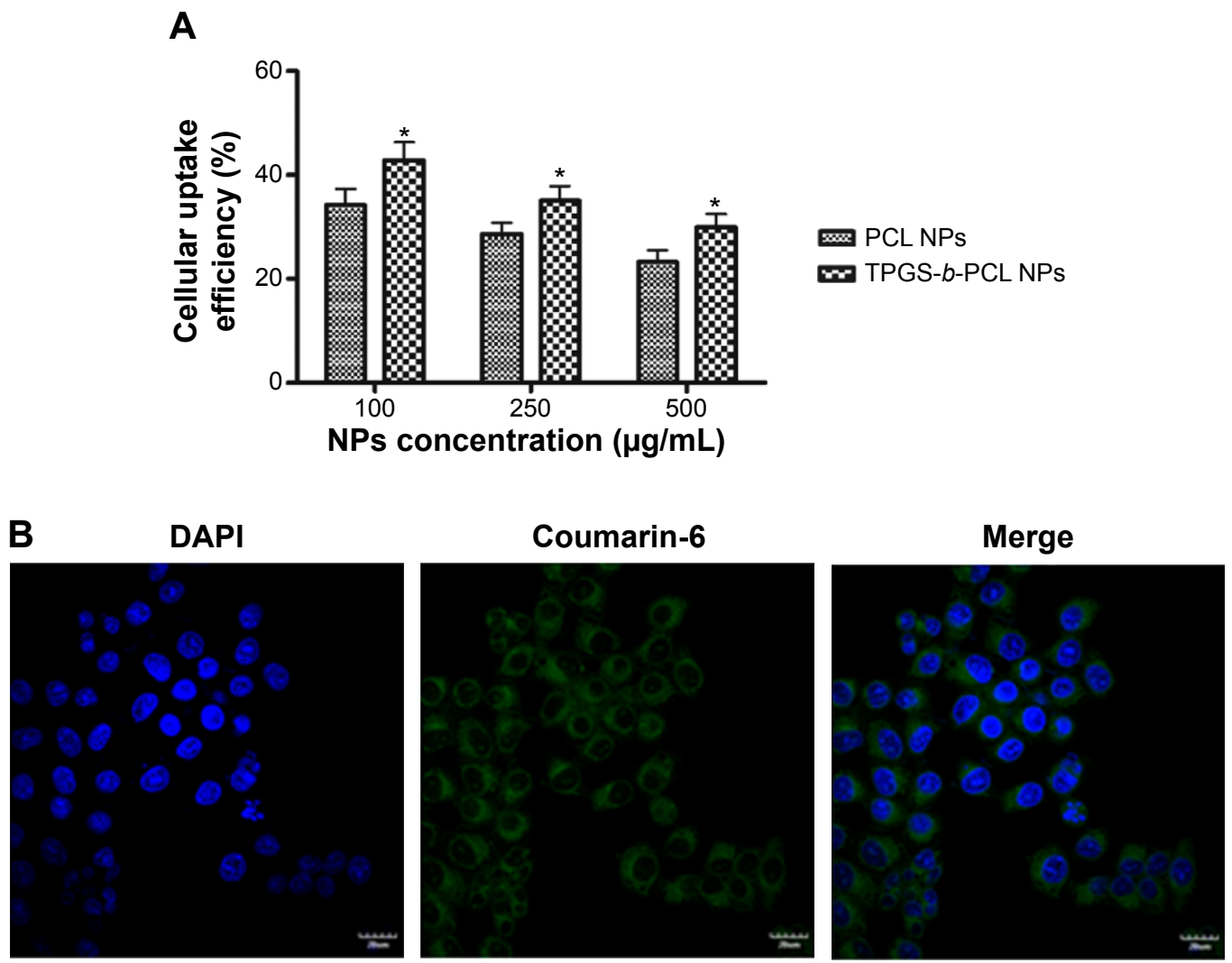

Figure 9 Cellular uptake of coumarin-6-loaded PCL NPs and TPGS-b-PCL NPs after incubated with HeLa cells.

Notes: (A) Uptake efficiency of coumarin-6-loaded PCL NPs and TPGS-b-PCL NPs by HeLa cells $(* P<0.05)$; (B) confocal laser scanning microscopic images of HeLa cells after incubation with the coumarin-6-loaded TPGS-b-PCL NPs. Scale bar $10 \mu \mathrm{m}$.

Abbreviations: DAPI, 4',6-diamidino-2-phenylindole; NPs, nanoparticles; PCL, poly( $\varepsilon$-caprolactone); TPGS, D- $\alpha$-tocopheryl polyethylene glycol I000 succinate. 


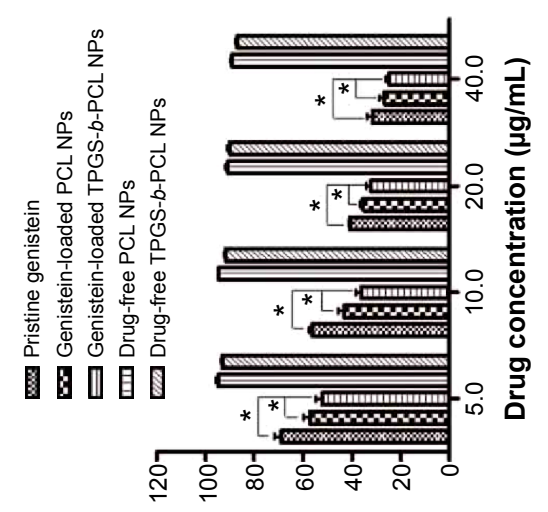

0

(\%) К‼!qอ!^ І|әว

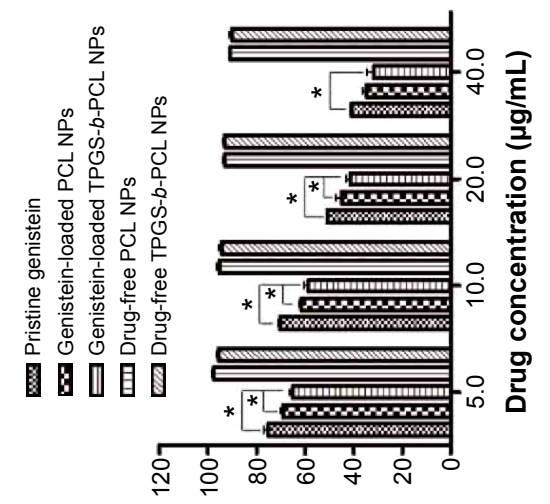

m

(\%) К‼!qe!ฺ ॥əכ

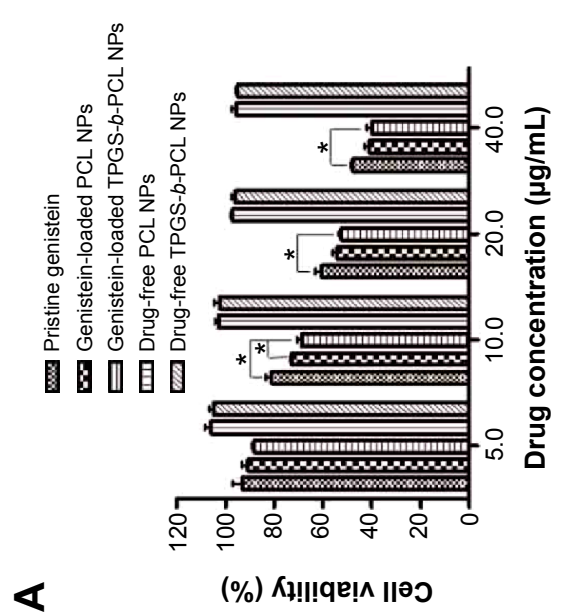

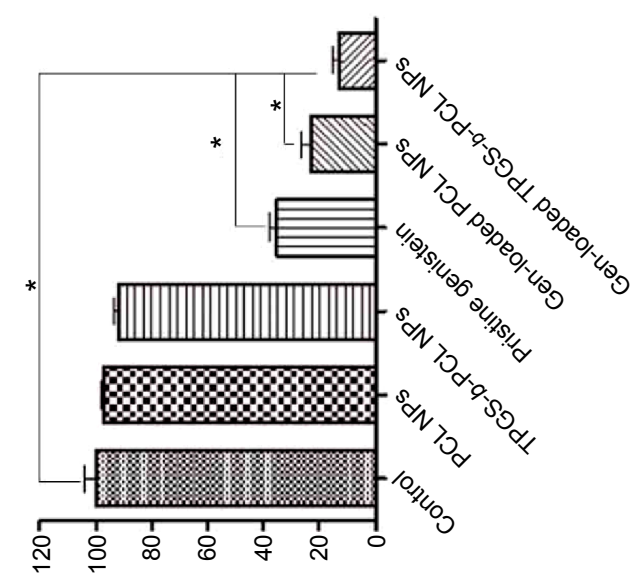

Ш

(\%) sə̣uoloo
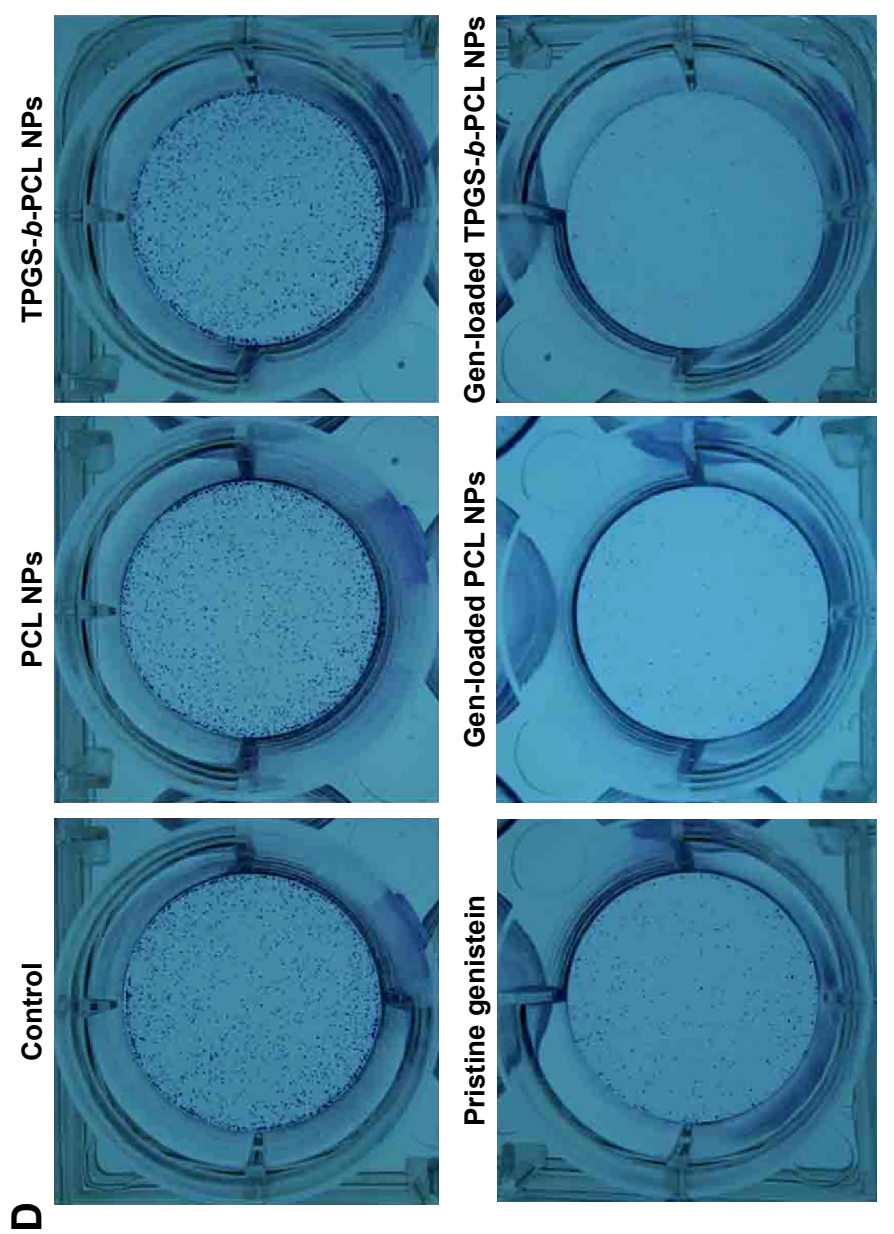

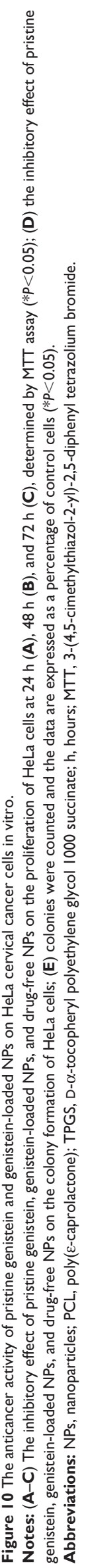


2) the cytotoxicity of the three genistein formulations were concentration- and incubation time-dependent; 3) the nanoformulations of genistein showed greater cytotoxicity than pristine genistein; 4) compared with pristine genistein and genistein-loaded PCL NPs, the genistein-loaded TPGS$b$-PCL NPs displayed significant advantages after 48 and 72 $\mathrm{h}$ of incubation. It could be seen from Figure 10B and C that the cell viability of HeLa decreased from $50.81 \%$ for pristine genistein, $44.85 \%$ for genistein-loaded PCL NPs, to $41.44 \%$ for genistein-loaded TPGS- $b$-PCL NPs (a 9.37\% and 3.41\% more effective in vitro therapeutic effect) after 48 hours of incubation and from $40.98 \%$ for pristine genistein, $35.38 \%$ for genisteinloaded PCL NPs, to $32.00 \%$ for genistein-loaded TPGS- $b$-PCL NPs (a $8.98 \%$ and $3.38 \%$ more effective in vitro therapeutic effect) after 72 hours of incubation at the drug concentration of $20.0 \mu \mathrm{g} / \mathrm{mL}$. Because of the cumulative release of genistein by NPs, both genistein-loaded PCL NPs and TPGS- $b$-PCL NPs exhibited a much higher cytotoxicity than did pristine genistein. Moreover, due to the degradation of TPGS- $b$-PCL NPs releasing TPGS, which has anticancer activity according to a previous study, genistein-loaded TPGS- $b$-PCL NPs showed a better antitumor effect than did genistein-loaded PCL NPs.

The antitumor effects of the three genistein formulations were further quantificated by the $\mathrm{IC}_{50}$ values from Figure 10 . Table 2 summarizes the $\mathrm{IC}_{50}$ values of HeLa cells incubated with pristine genistein, genistein-loaded PCL NPs and TPGS$b$-PCL NPs at final concentrations of 5.0, 10.0, 20.0, 30.0, $40.0,60.0$, and $80.0 \mu \mathrm{g} / \mathrm{mL}$ equivalent genistein for 24,48 , and 72 hours. It was shown that the $\mathrm{IC}_{50}$ values for $\mathrm{HeLa}$ cells were $31.3,23.9$, and $13.6 \mu \mathrm{g} / \mathrm{mL}$ for pristine genistein, $26.1,16.5$, and $7.4 \mu \mathrm{g} / \mathrm{mL}$ for genistein-loaded PCL NPs, and 24.3, 13.6, and $5.0 \mu \mathrm{g} / \mathrm{mL}$ for genistein-loaded TPGS- $b$-PCL NPs after treatment for 24, 48, and 72 hours, respectively. That is, drugs encapsulated by the NPs exhibited higher cytotoxicity. Furthermore, we could conclude that the in vitro antitumor effects of the three genistein formulations were in the following order: genistein-loaded TPGS- $b$-PCL NPs $>$ genistein-loaded PCL NPs $>$ pristine genistein.

Table $2 \mathrm{IC}_{50}$ values of pristine genistein, genistein-loaded PCL NPs, and genistein-loaded TPGS-b-PCL NPs on human cervical carcinoma cell line HeLa after 24, 48, and 72 hours of incubation

\begin{tabular}{|c|c|c|c|}
\hline \multirow{2}{*}{$\begin{array}{l}\text { Incubation } \\
\text { time (hours) }\end{array}$} & \multicolumn{3}{|c|}{$I C_{50}(\mu \mathrm{g} / \mathrm{mL})$} \\
\hline & $\begin{array}{l}\text { Pristine } \\
\text { genistein }\end{array}$ & PCL NPs & TPGS-b-PCL NPs \\
\hline 24 & $31.3 \pm 3.2$ & $26.1 \pm 1.9$ & $24.3 \pm 0.6$ \\
\hline 48 & $23.9 \pm 1.1$ & $16.5 \pm 2.7$ & $13.6 \pm 1.4$ \\
\hline 72 & $13.6 \pm 0.9$ & $7.4 \pm 0.8$ & $5.0 \pm 0.8$ \\
\hline
\end{tabular}

Abbreviations: $\mathrm{IC}_{50}$, the half maximal inhibitory concentration; NPs, nanoparticles; $\mathrm{PCL}$, poly( $\varepsilon$-caprolactone); TPGS, D- $\alpha$-tocopheryl polyethylene glycol 1000 succinate.
In order to further examine the cytotoxicity and growth inhibitory effects of the three genistein formulations on HeLa cells, colony formation assay was carried out. As shown in Figure 10D and E, all the genistein formulations significantly impaired the cell colony formation, with genistein-loaded TPGS- $b$-PCL NPs having the strongest effect, while the drugfree NPs showing no obvious effect. This result is consistent with that of the cytotoxicity assay.

\section{Anticancer efficacy of genistein in vivo}

To compare the antitumor activity of genistein-loaded TPGS$b$-PCL nanoformulation with that of the pristine genistein in vivo, HeLa cells were subcutaneously inoculated into the right flank of female BALB/c nude mice. When the tumor xenografts were touchable, mice were divided to three groups ( $\mathrm{n}=5$ for each group) and injected intraperitoneally every 2 days with saline, pristine genistein, genistein-loaded PCL NPs, or genistein-loaded TPGS- $b$-PCL NPs at the genistein dose of $50 \mathrm{mg} / \mathrm{kg}$. As shown in Figure 11A, all the three genistein formulations significantly decreased the growth of HeLa tumor in vivo, with genistein-loaded TPGS- $b$ PCL NPs being more effective in comparison with pristine genistein and genistein-loaded PCL NPs. Moreover, all the three genistein formulations were well tolerated without significant impact on the mice body weight (Figure 11B). After treatment for 16 days, the mice were humanely killed and tumors were excised and weighted. As shown in Figure 11C, compared with saline group, the tumor weight in all the three genistein formulations-treated groups was significantly reduced, and the mice treated with genistein-loaded TPGS$b$-PCL NPs exhibited the lightest tumor weight. Furthermore, the images of tumors from each group also indicated the advantages of genistein-loaded TPGS- $b$-PCL NPs versus pristine genistein and genistein-loaded PCL NPs in repressing tumor growth (Figure 11D). All animal care and procedures for animal experiments were with approval from the Administrative Committee on Animal Research in Tsinghua University.

\section{Conclusion}

In this research, biodegradable TPGS- $b$-PCL NPs entrapping genistein were prepared using a modified nanoprecipitation method to treat HeLa cervical carcinoma in vitro and in vivo. The obtained genistein-loaded TPGS- $b$-PCL NPs showed smaller size, higher DL and EE, faster drug release rate, and higher cellular uptake efficiency and cytotoxicity than genistein-loaded PCL NPs. All of these could be attributed to the introduction of the TPGS component into the PCL polymer, which made the PCL polymer more suitable 
A

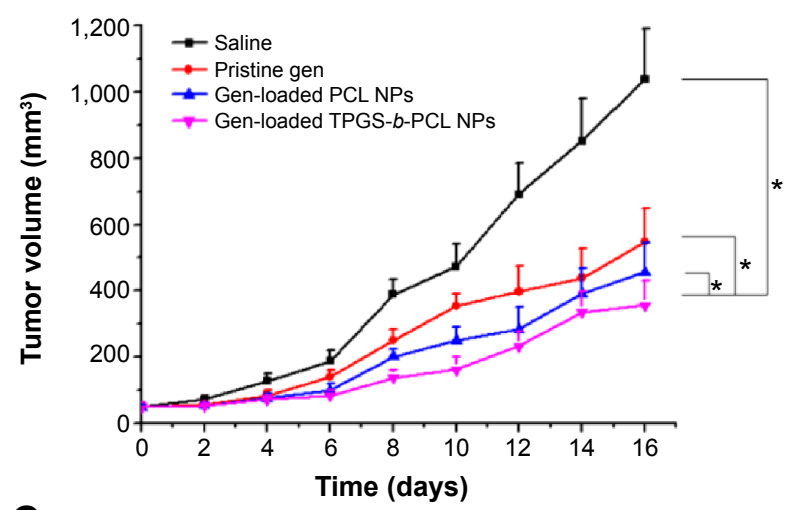

C

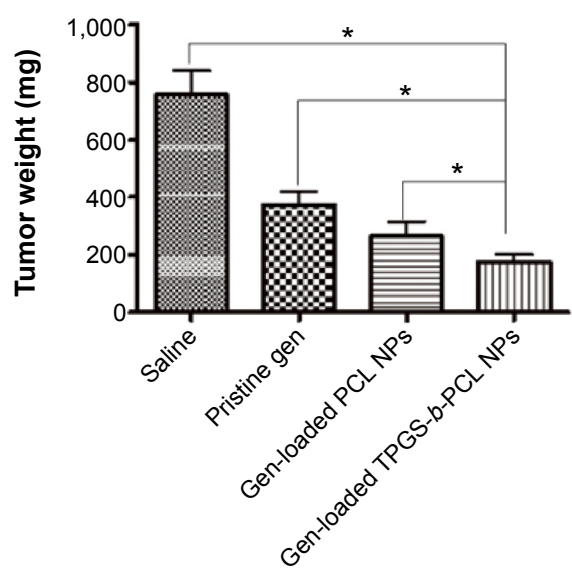

B

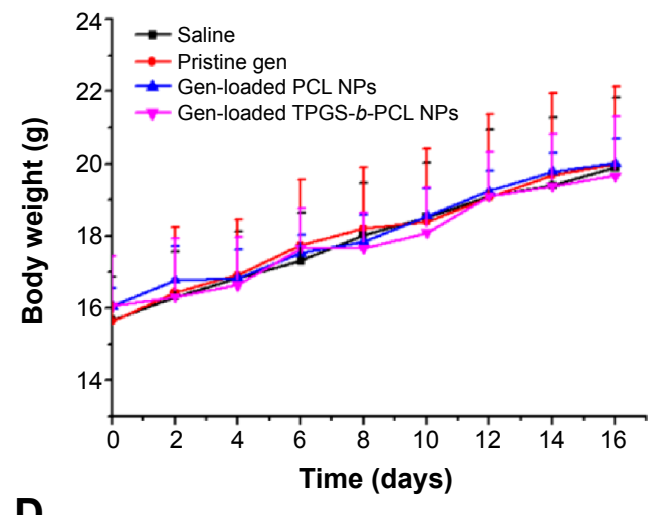

D

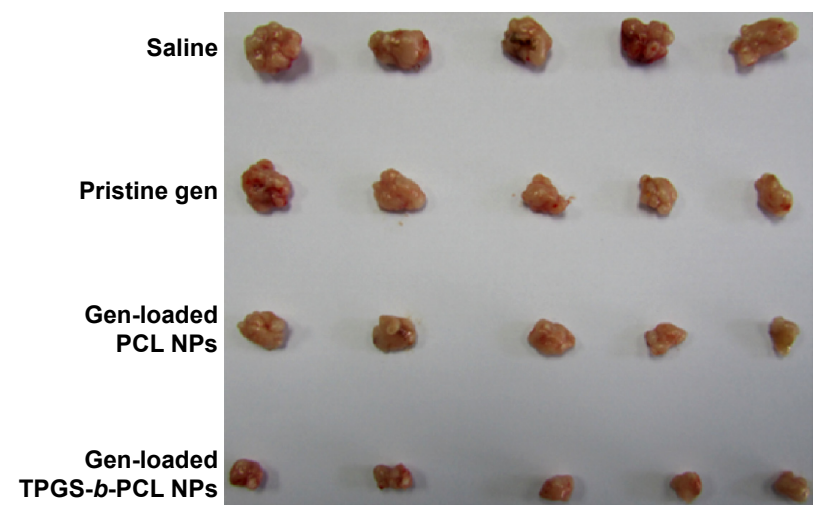

Figure I I Antitumor effect of genistein formulated in TPGS-b-PCL in comparison with pristine genistein and genistein-loaded PCL NPs ( $n=5$ ).

Notes: (A) Tumor growth curve of the BALB/c nude mice bearing HeLa cells xenograft after administration $\left({ }^{*} P<0.05\right)$; (B) body weight after administration; (C) weight of the tumor from each group taken out from the sacrificed mice at the end of the study $(* P<0.05)$; (D) image of tumor from each group taken out from the sacrificed mice at the end of the study.

Abbreviations: NPs, nanoparticles; PCL, poly( $\varepsilon$-caprolactone); TPGS, D- $\alpha$-tocopheryl polyethylene glycol I000 succinate.

for drug delivery. Compared with pristine genistein, the genistein-loaded TPGS- $b$-PCL NPs were more effective in the suppression of HeLa cell growth both in vitro and in vivo. Furthermore, both the genistein formulations did not show any significant effect on the mice body weight. Therefore, the prepared genistein-loaded TPGS- $b$-PCL NPs are an excellent low-toxic aqueous formulation of genistein with improved anticancer activity, which may have potential application in cervical cancer therapy.

\section{Acknowledgments}

The authors are grateful for financial support from the National Natural Science Foundation of China (Numbers 31270019, 51203085), China Postdoctoral Science Foundation (Number 2012M520242), Natural Science Foundation of Guangdong Province (Numbers S2012040006820, S2012010010046), Science, Technology and Innovation Commission of Shenzhen Municipality (Numbers JCYJ20120616213729920, KQC201105310021A,
JCYJ20120614191936420, JC201005270308A), and Program for New Century Excellent Talents in University (NCET-11-0275).

\section{Disclosure}

The authors report no conflicts of interest in this work.

\section{References}

1. Jemal A, Bray F, Center MM, Ferlay J, Ward E, Forman D. Global cancer statistics. CA Cancer J Clin. 2011;61:69-90.

2. Li-Weber M. Targeting apoptosis pathways in cancer by Chinese medicine. Cancer Lett. 2013;332:304-312.

3. Buer CS, Imin N, Djordjevic MA. Flavonoids: new roles for old molecules. J Integr Plant Biol. 2010;52:98-111.

4. Spagnuolo C, Russo M, Bilotto S, Tedesco I, Laratta B, Russo GL. Dietary polyphenols in cancer prevention: the example of the flavonoid quercetin in leukemia. Ann N Y Acad Sci. 2012;1259:95-103.

5. Li QS, Li CY, Li ZL, Zhu HL. Genistein and its Synthetic Analogs as Anticancer Agents. Anticancer Agents Med Chem. 2012;12:271-281.

6. Ullah MF, Ahmad A, Zubair H, et al. Soy isoflavone genistein induces cell death in breast cancer cells through mobilization of endogenous copper ions and generation of reactive oxygen species. Mol Nutr Food Res. 2011;55:553-559. 
7. Hussain A, Harish G, Prabhu SA, et al. Inhibitory effect of genistein on the invasive potential of human cervical cancer cells via modulation of matrix metalloproteinase-9 and tissue inhibitiors of matrix metalloproteinase-1 expression. Cancer Epidemiol. 2012;36:e387-e393.

8. Li HQ, Luo Y, Qiao CH. The mechanisms of anticancer agents by genistein and synthetic derivatives of isoflavone. Mini Rev Med Chem. 2012;12:350-362.

9. Ouyang G, Yao L, Ruan K, Song G, Mao Y, Bao S. Genistein induces G2/M cell cycle arrest and apoptosis of human ovarian cancer cells via activation of DNA damage checkpoint pathways. Cell Biol Int. 2009;33:1237-1244.

10. Qi W, Weber CR,Wasland K, Savkovic SD. Genistein inhibits proliferation of colon cancer cells by attenuating a negative effect of epidermal growth factor on tumor suppressor FOXO3 activity. BMC Cancer. 2011;11:219.

11. Kim SH, Lee SC, Song YS. Involvement of both extrinsic and intrinsic apoptotic pathways in apoptosis induced by genistein in human cervical cancer cells. Ann N Y Acad Sci. 2009;1171:196-201.

12. Guo G, Fu S, Zhou L, et al. Preparation of curcumin loaded poly ( $\varepsilon$-caprolactone)-poly (ethylene glycol)-poly ( $\varepsilon$-caprolactone) nanofibers and their in vitro antitumor activity against Glioma 9L cells. Nanoscale. 2011;3:3825-3832.

13. Li X, Zhang Z, Li J, Sun S, Weng Y, Chen H. Diclofenac/biodegradable polymer micelles for ocular applications. Nanoscale. 2012;4 4667-4673.

14. Parrott MC, DeSimone JM. Relieving PEGylation. Nat Chem. 2012; 4:13-14.

15. Tomasina J, Lheureux S, Gauduchon P, Rault S, Malzert-Freon A Nanocarriers for the targeted treatment of ovarian cancers. Biomaterials 2013;34:1073-1101.

16. Mi Y, Liu X, Zhao J, Ding J, Feng SS. Multimodality treatment of cancer with herceptin conjugated, thermomagnetic iron oxides and docetaxel loaded nanoparticles of biodegradable polymers. Biomaterials 2012;33:7519-7529.

17. Mei L, Zhang Z, Zhao L, et al. Pharmaceutical nanotechnology for oral delivery of anticancer drugs. Adv Drug Deliv Rev. 2013;65:880-890.

18. Huang L, Chen $\mathrm{H}$, Zheng $\mathrm{Y}$, et al. Nanoformulation of d- $\alpha$-tocopheryl polyethylene glycol 1000 succinate-b-poly ( $\varepsilon$-caprolactone-ranglycolide) diblock copolymer for breast cancer therapy. Integr Biol (Camb). 2011;3:993-1002.

19. Gao X, Wang B, Wei X, et al. Anticancer effect and mechanism of polymer micelle-encapsulated quercetin on ovarian cancer. Nanoscale. 2012;4:7021-7030.

20. Gong C, Deng S, Wu Q, et al. Improving antiangiogenesis and antitumor activity of curcumin by biodegradable polymeric micelles. Biomaterials. 2013;34:1413-1432.

21. Zhang Z, Mei L, Feng SS. Vitamin E D- $\alpha$-tocopheryl polyethylene glycol 1000 succinate-based nanomedicine. Nanomedicine (London). 2012;7:1645-1647.

22. Youk HJ, Lee E, Choi MK, et al. Enhanced anticancer efficacy of $\alpha$-tocopheryl succinate by conjugation with polyethylene glycol. $J$ Control Release. 2005;107:43-52.
23. Collnot EM, Baldes C, Schaefer UF, Edgar KJ, Wempe MF, Lehr CM. Vitamin E TPGS P-glycoprotein inhibition mechanism: influence on conformational flexibility, intracellular ATP levels, and role of time and site of access. Mol Pharm. 2010;7:642-651.

24. Guo Y, Luo J, Tan S, Otieno BO, Zhang Z. The applications of Vitamin E TPGS in drug delivery. Eur J Pharm Sci. 2013;49:175-186.

25. Acharya S, Sahoo SK. PLGA nanoparticles containing various anticancer agents and tumour delivery by EPR effect. Adv Drug Deliv Rev. 2011;63:170-183.

26. Zeng X, Tao W, Mei L, Huang L, Tan C, Feng SS. Cholic acidfunctionalized nanoparticles of star-shaped PLGA-vitamin E TPGS copolymer for docetaxel delivery to cervical cancer. Biomaterials. 2013; 34:6058-6067.

27. Valencia PM, Hanewich-Hollatz MH, Gao W, et al. Effects of ligands with different water solubilities on self-assembly and properties of targeted nanoparticles. Biomaterials. 2011;32:6226-6233.

28. Gou M, Men K, Shi H, et al. Curcumin-loaded biodegradable polymeric micelles.

29. Yan F, Zhang C, Zheng Y, et al. The effect of poloxamer 188 on nanoparticle morphology, size, cancer cell uptake, and cytotoxicity. Nanomedicine. 2010;6:170-178.

30. Mi Y, Liu Y, Feng SS. Formulation of docetaxel by folic acid-conjugated d- $\alpha$-tocopheryl polyethylene glycol succinate 2000 (Vitamin E TPGS $2 \mathrm{k}$ ) micelles for targeted and synergistic chemotherapy. Biomaterials. 2011;32:4058-4066.

31. Mi Y, Zhao J, Feng SS. Targeted co-delivery of docetaxel, cisplatin and herceptin by vitamin E TPGS-cisplatin prodrug nanoparticles for multimodality treatment of cancer. J Control Release. 2013;169:185-192.

32. Zhang Y, Tang L, Sun L, et al. A novel paclitaxel-loaded poly ( $\varepsilon$-caprolactone)/poloxamer 188 blend nanoparticle overcoming multidrug resistance for cancer treatment. Acta Biomater. 2010;6: 2045-2052.

33. Albanese A, Tang PS, Chan WC. The effect of nanoparticle size, shape, and surface chemistry on biological systems. Annu Rev Biomed Eng. 2012;14:1-16

34. Petros RA, DeSimone JM. Strategies in the design of nanoparticles for therapeutic applications. Nat Rev Drug Discov. 2010;9:615-627.

35. Walkey CD, Olsen JB, Guo H, Emili A, Chan WC. Nanoparticle size and surface chemistry determine serum protein adsorption and macrophage uptake. J Am Chem Soc. 2012;134:2139-2147.

36. Kulkarni SA, Feng SS. Effects of particle size and surface modification on cellular uptake and biodistribution of polymeric nanoparticles for drug delivery. Pharm Res. 2013;30(10):2512-2522.

37. Van Eerdenbrugh B, Vermant J, Martens JA, et al. A screening study of surface stabilization during the production of drug nanocrystals J Pharm Sci. 2009;98:2091-2103.

38. de Planque MR, Aghdaei S, Roose T, Morgan H. Electrophysiological characterization of membrane disruption by nanoparticles. ACS Nano. 2011;5:3599-3606.
International Journal of Nanomedicine

\section{Publish your work in this journal}

The International Journal of Nanomedicine is an international, peerreviewed journal focusing on the application of nanotechnology in diagnostics, therapeutics, and drug delivery systems throughout the biomedical field. This journal is indexed on PubMed Central, MedLine, CAS, SciSearch $®$, Current Contents $\AA /$ Clinical Medicine,

\section{Dovepress}

Journal Citation Reports/Science Edition, EMBase, Scopus and the Elsevier Bibliographic databases. The manuscript management system is completely online and includes a very quick and fair peer-review system, which is all easy to use. Visit http://www.dovepress.com/ testimonials.php to read real quotes from published authors. 